\title{
Radiação solar e distribuição vertical de área foliar em floresta - Reserva Biológica do Cuieiras - ZF2, Manaus
}

\author{
Arí de O. MARQUES FILHO ${ }^{1}$, Ricardo G. DALLAROSA ${ }^{1}$, Vanusa Bezerra PACHÊCO ${ }^{2}$
}

\begin{abstract}
RESUMO
Neste estudo, a distribuição vertical de área foliar em floresta é investigada em conexão com o regime de radiação, usando as medidas de radiação solar realizadas no período de julho a novembro de 2001, na Reserva Biológica do Cuieiras - Manaus -ZF2, $\mathrm{km} 14 \mathrm{e} \mathrm{km} \mathrm{34,} \mathrm{na} \mathrm{Amazônia} \mathrm{Central.} \mathrm{Técnicas} \mathrm{experimentais} \mathrm{de} \mathrm{amostragem} \mathrm{de} \mathrm{radiação} \mathrm{no} \mathrm{interior} \mathrm{de} \mathrm{coberturas} \mathrm{vegetais} \mathrm{de}$ grande porte são utilizadas, com dispositivos de suporte móveis constituídos por reticulados modulares, que permitem a disposição dos sensores de radiação em diferentes níveis de uma mesma vertical no interior da cobertura. Inversão de modelos radiativos em coberturas vegetais densas permite as análises sobre a distribuição vertical de área foliar. A variabilidade espacial de área foliar (IAF, função a(z)) é estabelecida para os dois sítios experimentais a partir de medidas de radiação solar, individualizadas em três verticais em cada um desses locais. O índice de área foliar total médio (IAF) da vegetação local para o sítio experimental do $\mathrm{km} 14$ alcançou o valor de 6,4 e para o sítio experimental do $\mathrm{km} 34$ o valor de 6,1. Uma análise comparativa é desenvolvida sobre distribuições verticais de área foliar obtidas em sítios experimentais da Amazônia, usando o mesmo sistema de medidas de radiação solar.
\end{abstract}

\section{PALAVRAS CHAVES}

Índice de área foliar, radiação solar, floresta, Amazônia.

\section{Solar radiation and vertical leaf area distribution in forest - Reserva Biológica do Cueiras - ZF02, Manaus}

\begin{abstract}
In this work, the vertical leaf area distribution is investigated in connection with radiation regime, using the measurements of solar radiation made from July to November 2001 at the experimental site of Reserva Biológica do Cuieiras, Manaus, ZF2-km 14 and km 34, in Central Amazonia. A sampling technique is used for the radiation measurements inside two tall canopy covers, having a mobile support apparatus, made up of modular frame, which allows placement of the radiation sensors at different beights along a vertical line within the canopy. Inversion of the solar radiation physical model allowed a determination of the leaf area density. Spatial variability of leaf area (LAI, function a(z)) is established for the two experimental sites from the measurements of solar radiation in three verticals on each site. The average values of leaf area index (LAI) for the local vegetation at km 14 and at km 34-ZF2 were 6,4 and 6,1, respectively. A comparative analysis is developed concerning the vertical distributions of leaf area obtained for different experimental sites in Amazonia, using the same solar radiation measurement system.
\end{abstract}

KEYWORDS

Leaf area index, solar radiation, forest, Amazonia.

${ }^{1}$ Instituto Nacional de Pesquisas da Amazônia - INPA, Cx. Postal 478, 69011-970 Manaus, AM, Brasil

${ }^{2}$ Universidade Estadual do Amazonas - UEA, Parintins. Bolsista DTI do INPA. 


\section{ACTA \\ AMAZONICA}

RADIAÇÃO SOLAR E DISTRIBUICCÃO VERTICAL DE ÁREA FOLIAR EM

FLORESTA - RESERVA BIOLÓGICA DO CUIEIRAS - ZF2, MANAUS

\section{INTRODUÇÃO}

No domínio de estudos sobre clima e vegetação, os intercâmbios de energia e de massa entre coberturas vegetais e a atmosfera têm reguladores próprios na vegetação associados aos aspectos estruturais e fisiológicos que a caracterizam. As relações energéticas que originam e sustentam esses intercâmbios são dirigidos por uma fonte primária de radiação solar externa e independente, complementada pela radiação 'interna' de onda longa dos elementos vegetais e do solo subjacente à cobertura.

A atenuação da radiação solar em meio vegetal é um fenômeno físico de dupla utilidade nos limites pretendidos desta abordagem: por um lado, o conhecimento de seu perfil vertical no interior de uma cobertura vegetal permite a definição da distribuição de energia disponível nos diferentes estratos da vegetação e por conseqüência dos perfis verticais das fontes de calor e de vapor d'água na cobertura; por outro lado, a estreita dependência existente entre essa atenuação e a distribuição espacial dos elementos vegetais constitui a base de apoio para uma grande parte dos métodos indiretos de caracterização de uma cobertura vegetal, especialmente na sua função de densidade de área foliar (distribuição vertical e variabilidade horizontal) e no seu índice de área foliar, IAF.

Modelos físicos explicitados de forma analítica reúnem os conceitos teóricos sobre a radiação solar em meio vegetal (Nilson, 1971; Ross, 1981; Myneni et al., 1989; Marques Filho, 1992) e são apropriados à inversão matemática para a obtenção das características principais da vegetação (índice de área foliar, IAF; distribuição vertical de densidade de área foliar) (Welles \& Norman, 1991; Andrieu \& Baret, 1993).

Estudos dessa natureza têm sido realizados em coberturas vegetais de médio e grande porte na Amazônia, constituindose de determinações do IAF e da distribuição vertical da função a(z) (densidade de área foliar) por métodos indiretos baseados na penetração de radiação solar no meio vegetal (Honzák et al., 1996; Wandelli \& Marques Filho, 1999). Tais determinações constituem importantes alternativas aos métodos destrutivos e trabalhosos de medida direta de toda área foliar existente numa área escolhida (McWilliam et al., 1993).

As variações temporais da radiação solar e a distribuição vertical de área foliar em florestas foram medidas e estudadas com o suporte de torre metálica que sobressai acima das copas das árvores (Marques Filho, 1997). As dificuldades principais em tais experimentos estão sempre associadas à proximidade inevitável entre os sensores e os elementos estruturais da torre (afastamentos normais de $5 \mathrm{a} 6 \mathrm{~m}$ ). Nas interações radiação solar - vegetação, a presença da torre, com seus patamares regularmente distribuídos na vertical, incorpora superfícies adicionais (estranhas à vegetação) que interceptam radiação e cujos efeitos sobre as medidas não podem ser separados facilmente da atenuação natural da radiação imposta pela vegetação. O desenvolvimento de dispositivo alternativo de medidas de radiação solar no interior da vegetação, que minimiza essas dificuldades experimentais, foi apresentado e aplicado por Marques Filho \& Dallarosa $(2000,2001)$.
Nesse contexto, o presente estudo tem os seguintes objetivos: analisar o comportamento da radiação solar no interior de floresta, enfocando a sua variabilidade espaçotemporal em associação com a distribuição de área foliar local; inverter modelo físico do regime de radiação solar em meio vegetal para a derivação da função a(z) de densidade de área foliar média para a cobertura e comparar os resultados obtidos com a mesma metodologia para diferentes sítios experimentais na Região Amazônica.

\section{MATERIAL E MÉTODOS}

\section{A - Área de estudo}

Sítio/Vegetação - Os sítios investigados são representados por duas áreas de platô de uma mesma toposseqüência, distantes entre si $10 \mathrm{~km}$ numa linha reta, imersos numa grande área de floresta primária, na Reserva Biológica do Cuieiras (22735 ha), do Instituto Nacional de Pesquisas da Amazônia, situada cerca de $60 \mathrm{~km}$ a noroeste de Manaus. $O$ acesso à reserva é feito por via rodoviária, através de uma estrada de terra vicinal (ZF-2) e os platôs acham-se próximos ao $\mathrm{km} 14 \mathrm{e} \mathrm{km} 34$ da mesma. Em cada platô existe uma torre micrometeorológica, $\operatorname{com} 41,5$ $\mathrm{m}$ e $50 \mathrm{~m}$ de altura, cujas localizações correspondem, respectivamente, a $02^{\circ} 35^{\prime} 21^{\prime \prime} \mathrm{S} ; 60^{\circ} 06^{\prime} 54^{\prime \prime} \mathrm{W}$; altitude $140 \mathrm{me}$ $02^{\circ} 36^{\prime} 33^{\prime \prime} \mathrm{S} ; 60^{\circ} 12^{\prime} 33^{\prime \prime} \mathrm{W}$; altitude $130 \mathrm{~m}$. O relevo é constituído por diversos platôs intercalados por vales onde aparecem vários igarapés, compondo uma rica rede de escoamento; o solo é do tipo latossolo amarelo álico, de textura argilosa, com boa drenagem (Chauvel, 1982).

A vegetação é típica de floresta tropical primária não perturbada, bastante diversificada no aspecto florístico, cujo dossel alcança uma altura entre $30-40 \mathrm{~m}$, com a ocorrência de cerca de 324 espéciese 174 gêneros por ha (Jardim \& Hosokawa, 1986/87). Oliveira et al. (2002), realizaram recente inventário no platô do $\mathrm{km} \mathrm{34}$, identificando apenas indivíduos com diâmetro à altura do peito (DAP) igual ou superior a $10 \mathrm{~cm}$, tendo encontrado 670 indivíduos, distribuídos em 48 famílias, 133 gêneros e 245 espécies.

Microclima - O clima local, obtido de uma série de dados (1980/1990) de uma estação climatológica do tipo principal, localizada no km 14 da ZF-2, é típico da Amazônia Central com temperaturas elevadas, uma boa abundância de chuvas ao longo do ano e uma curta estação seca. Os índices pluviométricos registram totais médios mensais superiores a $150 \mathrm{~mm}$ em pelo menos nove meses do ano (entre outubro e junho) e apenas três meses com valores inferiores (julho a setembro), tendo o mês de agosto apresentado o menor índice $(99 \mathrm{~mm})$. A Zona de Convergência Intertropical (ZCIT) tem um importante papel no regime pluviométrico da região por deslocar para ela grandes quantidades da umidade produzida no oceano Atlântico. A umidade relativa média mensal foi sempre superior a $80 \%$ e seguiu, conforme o esperado, o comportamento 


\section{ACTA AMAZONICA}

RADIAÇÃO SOLAR E DISTRIBUIÇÃO VERTICAL DE ÁREA FOLIAR EM FLORESTA - RESERVA BIOLÓGICA DO CUIEIRAS - ZF2, MANAUS sazonal da precipitação. As temperaturas médias mensais variaram entre $25,2^{\circ} \mathrm{C}$ (julho) e $26,7^{\circ} \mathrm{C}$ (novembro). As máximas oscilaram entre os $31,3^{\circ} \mathrm{C}$ em fevereiro e $33,2^{\circ} \mathrm{C}$ em setembro, enquanto as mínimas estiveram entre $21,5^{\circ} \mathrm{C}$ em julho e 23,0 ${ }^{\circ} \mathrm{C}$ em março. A ocorrência de valores extremos de temperatura nos meses secos, tanto máximos como mínimos, pode ser explicada pela baixa nebulosidade da estação, que permite maior quantidade de radiação solar à superfície durante o dia e uma maior perda radiativa durante a noite, associada com a presença mais freqüente de sistemas frontais provenientes do sul, durante o período.

\section{B - Medidas de radiação}

Usualmente, tem-se adotado a utilização de mastros ou torres de estrutura metálica para a fixação dos instrumentos de medida. Essa estrutura, entretanto, interage com o ambiente, afetando as grandezas medidas de forma tanto mais significativa quanto maior for a influência determinada pela estrutura. Além disso, a dificuldade de deslocamento desses sistemas para a realização de medidas em várias verticais representa um fator limitante ao procedimento de múltipla amostragem. Assim sendo, foi criado um novo tipo de sistema que procura aproveitar o suporte natural representado pelas grandes árvores no sentido de suspender estruturas leves, flexíveis e modulares, formando um reticulado vertical de sustentação por cabos de aço, unindo seções paralelas (previamente montadas) e servindo finalmente de suporte aos sensores dispostos segundo um mesmo eixo vertical. Esse sistema, detalhadamente descrito por Marques Filho \& Dallarosa (2000) e reproduzido na figura 1 , foi utilizado neste trabalho. No contexto desse novo sistema de medidas, a escolha dos pontos amostrais dá-se ao acaso, limitando-se apenas à existência dos suportes de sustentação mais adequados representados pelas grandes árvores.

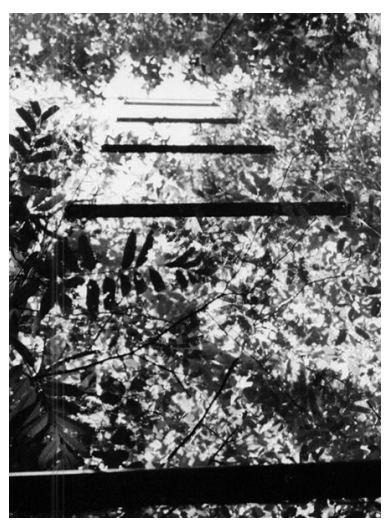

A

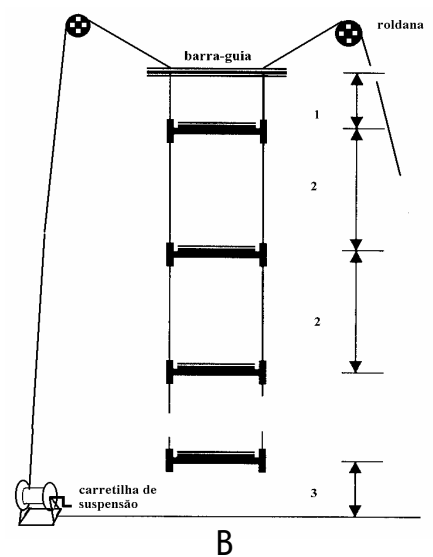

B
Figura 1 - Fotografia do sistema de medidas (reticulado) (a); Esquema de representação do suporte reticulado utilizado para a distribuição dos sensores de medidas de radiação no interior do dossel: (b) (Marques Filho \& Dallarosa, 2000).
As medidas de radiação solar em perfis sob a vegetação, utilizadas no modelo de inversão matemática, foram realizadas por um sistema automático de aquisição de dados (datalogger 21X, Campbell Scientific Inc, England) disposto junto ao solo. Os sensores utilizados foram solarímetros tubulares (Delta-T Devices Ltd, England) com superfície retangular de $2 \mathrm{~cm}$ x 100 cm e sensibilidade à radiação de comprimentos de onda entre 0,3 e 3,0 $\mu \mathrm{m}$ (intervalo característico do espectro solar), cujas medidas foram compostas a partir de varreduras dos sensores em intervalos de dez segundos, com subseqüentes integrações e cálculo de médias a intervalos de 20 minutos, no período diurno entre 06:00 às 18:00 horas (hora local). As medidas de radiação solar acima das copas das árvores foram geradas pelo mesmo tipo de sensor (solarímetro tubular), instalado no topo da torre micrometeorológica.

O conjunto total de medidas representa a distribuição de radiação solar acima e no interior da vegetação (perfis de radiação solar) em verticais distintas (designação usada ao longo do texto para indicar as linhas verticais de amostragem) situadas nas proximidades das torres metálicas existentes na área de estudo, sendo destinadas três verticais para cada um dos sítios experimentais. Os períodos de funcionamento do dispositivo em cada vertical foram os seguintes:

ZF2 - km 14

Primeira vertical, de 29/08 a 12/09/2001.

Segunda vertical, de 13/09 a 26/09/2001.

Terceira vertical, de 26/09 a 10/10/2001.

ZF2 - km 34

Primeira vertical, de 26/07 a 10/08/2001.

Segunda vertical, de 15/08 a 21/08/2001.

Terceira vertical, de 11/10 a 21/11/2001.

\section{C - Modelo de radiação solar e método de obtenção da densidade de área foliar}

A atenuação da radiação solar em meio vegetal é resultado primário da quantidade de elementos vegetais que interceptam os raios solares em seu percurso desde o topo até uma determinada altura no interior da vegetação. A orientação e a distribuição espacial desses elementos são outros fatores intervenientes que admitem e determinam configurações especiais da arquitetura da cobertura, responsáveis pelo grau de complexidade encontrado no estabelecimento e na obtenção das soluções de equações que governam o regime de radiação em cada tipo de cobertura. Em vegetação densa algumas hipóteses sobre a variação desses fatores (distribuição espacial aleatória e orientação aleatória das normais dos elementos vegetais são os exemplos mais comuns) simplificam o aparato matemático necessário. Nesses casos, a quantificação de área foliar existente em cada zona da vegetação obtida de forma indireta a partir de medidas de radiação torna-se factível 


\section{ACTA \\ AMAZONICA}

RADIAÇÃO SOLAR E DISTRIBUICCÃO VERTICAL DE AREA FOLIAR EM

FLORESTA - RESERVA BIOLÓGICA DO CUIEIRAS - ZF2, MANAUS e tem como ponto de partida o estabelecimento de uma relação (modelos físicos ou matemáticos) entre os fluxos de radiação solar e a área foliar acumulada (variação vertical preponderante), arranjados em equações do tipo, (Marques Filho \& Dallarosa, 2000,2001),

$$
\alpha_{t}=\alpha_{t}(A)=\frac{F_{t}(A)}{F_{t}(A=0)}
$$

onde,

$$
\begin{aligned}
& \mathrm{F}=\text { fluxo de radiação }\left(\mathrm{W} \cdot \mathrm{m}^{-2}\right) \\
& \mathrm{A}=\text { área foliar acumulada (adimensional) } \\
& \alpha=\text { fluxo relativo de radiação, que é dado pela razão entre }
\end{aligned}
$$
o fluxo medido em uma altura $\mathrm{z}$ da vegetação e o fluxo no topo.

$\mathrm{t}=$ sub-índice que indica radiação total (direta + difusa).

Nessas equações, as variáveis A, $\alpha$ e F são dependentes da coordenada vertical e a omissão de tal dependência tem apenas o papel de focalizar as relações específicas que estão sendo apresentadas. Assim, com a subseqüente preparação do procedimento de inversão (expresso usualmente na forma de um algoritmo computacional), a função inversa, que permite a estimativa de área foliar acumulada para cada ponto de interesse de uma mesma vertical, é dada formalmente por (Marques Filho \& Dallarosa 2000,2001):

$\mathrm{A}=\mathrm{A}\left(\alpha_{\mathrm{t}}\right)$

sendo, o índice de área foliar acumulada na altura $\mathrm{z}$ dado por,

$$
A(z)=\int_{z}^{h} a(z) d z
$$

onde,

$$
\begin{aligned}
& \mathrm{a}=\text { densidade de área foliar }\left(\mathrm{m}^{2} \cdot \mathrm{m}^{-3}\right) \\
& \mathrm{z}=\operatorname{altura}(\mathrm{m}) \\
& \mathrm{h}=\operatorname{altura} \text { da vegetação }(\mathrm{m})
\end{aligned}
$$

A associação entre fluxos relativos de radiação solar $\alpha_{t,} \mathrm{e}$ função A(z) de área foliar acumulada, as definições particulares dos fluxos de radiação direta, difusa e complementar no interior da vegetação, o algoritmo básico de inversão de modelo de radiação e os valores adotados para os diferentes parâmetros, seguem no presente estudo a metodologia proposta por Wandelli \& Marques Filho (1999).
Os elementos de modelagem física da radiação solar em meio vegetal constituem a base de apoio para a inversão matemática expressa na equação 2 , com a associação final entre os fluxos relativos no interior da cobertura e as estimativas correspondentes de área foliar acumulada; o procedimento de inversão materializa-se na forma de um programa computacional desenvolvido em linguagem de programação estruturada Fortran 77 , e, do ponto de vista matemático, é simplesmente um procedimento numérico iterativo de inversão.

\section{D - Processamento dos dados}

As medidas de radiação solar de todo o período de observação são agrupadas em quatro classes de radiação externa (I, II, III, IV), partindo de eventos em que predomina radiação difusa (céu encoberto, classes I e II) e alcançando aquelas ocasiões em que a radiação direta é dominante (céu limpo, classes III e IV) (Marques Filho \& Dallarosa 2000,2001).

Esses agrupamentos de dados viabilizaram a representação condensada do conjunto total de informações acumuladas nas três verticais de observação em cada platô. O tratamento desses dados gerou as estimativas de densidade de área foliar para os diferentes pontos no interior da floresta, que, organizadas em classes, favorecem as interpretações posteriores.

Os perfis médios da função A(z) (apresentados a seguir nas tabelas 1 a 6) permitem a derivação numérica da função de densidade de área foliar a (z) para vários pontos na vertical a partir da expressão,

$\mathrm{a}\left(\mathrm{z}_{\mathrm{m}}\right)=-\mathrm{dA}\left(\mathrm{z}_{\mathrm{m}}\right) / \mathrm{dz}=\left[\mathrm{A}\left(\mathrm{z}_{1}\right)-\mathrm{A}\left(\mathrm{z}_{2}\right)\right] /\left(\mathrm{z}_{2}-\mathrm{z}_{1}\right)$

$$
\text { sendo } z_{\mathrm{m}} \text { o ponto intermediário das alturas } z_{1} \text { e } z_{2} \text {. }
$$

A função A(z) deve ser representativa para a vegetação de um dado local e no estudo atual foi estabelecida como o perfil médio das três verticais com um índice de área foliar (IAF) total dado pela média dos valores da função A(z) estimada junto ao solo. Com esse procedimento, as distribuições verticais de área foliar foram determinadas (e aparecem representadas nas Figuras 2 e 3). A função de distribuição vertical de área foliar a(z), determinada no estudo atual, é representativa da vegetação local em razão do número de pontos de observação num mesmo eixo vertical, especialmente na parte baixa da vegetação, e do número de verticais de medidas (três verticais em cada sítio). Uma descrição interessante para a função a(z) é desenvolvida na equação 4 , permitindo interpretações adequadas sobre as dificuldades inerentes à sua formação a partir de dados experimentais. Essa equação é da forma,

$$
a(z)=\frac{\text { Area foliar da camada }}{\text { Volume da camada }}=\frac{\sum_{1}^{n} a_{f m}(i) \cdot V_{\text {copa }}(i)}{V_{\text {camada }}}=\frac{\sum_{1}^{n} a_{f m}(i) \cdot A_{\text {copa }}(i) \cdot \Delta h}{A_{\text {camada } a . ~} \Delta h}
$$

onde,

$a_{\mathrm{fm}} \quad$ - densidade média de área foliar $\left(\mathrm{m}^{2} / \mathrm{m}^{3}\right) ;$ 


\section{ACTA AMAZONICA}

$\mathrm{V}_{\text {copa }}$ - volume da copa da árvore i na camada

$\mathrm{A}_{\text {copa }}$-área da copa da árvore i na camada

$\Delta \mathrm{h}$ - espessura da camada

n - número de árvores (copas) presentes na camada

Para densidades foliares iguais entre as copas das diferentes árvores de uma mesma camada, tem-se uma equação simplificada do tipo,

$a(z)=a_{f m}\left[\frac{\sum_{1}^{n} A_{\text {copa }}(i)}{A_{\text {camada }}}\right]=a_{f m} .($ fator de ocupação $)$

O fator de ocupação expressa, numa dada camada horizontal da vegetação situada na altura $z$, a razão entre o volume ocupado na camada pelas copas das árvores e o volume total da camada.

\section{RESULTADOS E DISCUSSÃO}

O conjunto de medidas de radiação e a inversão do modelo físico de radiação em cobertura vegetal, organizados e aplicados segundo as etapas descritas acima, conduzem à determinação dos perfis de área foliar acumulada $\mathrm{A}(\mathrm{z})$ nos três pontos experimentais estabelecidos para cada um dos dois sítios experimentais: $\mathrm{km} 14 \mathrm{e} \mathrm{km} 34 \mathrm{da} Z \mathrm{ZF} 2$. Os resultados associados às três verticais de medidas situadas no km 14 são apresentados nas tabelas 1, 2 e 3; os resultados correspondentes às três verticais de medidas no sítio experimental do km 34 são reunidos nas tabelas 4,5 e 6 .

Os valores médios do índice de área foliar total da cobertura em cada sítio variam em faixas de valores inferiores a 0,25 , em

Tabela 1 - Reserva do Cuieiras - ZF2, km 14; Primeira Vertical: 29 ago - 12 set 2001 . IAF calculado para cada classe de radiação, valores entre parênteses representam o desvio padrão.

\begin{tabular}{|c|c|c|c|c|c|}
\hline \multirow{3}{*}{$\begin{array}{l}\text { Nível de } \\
\text { medida } \\
(\mathrm{m}) \text { no } \\
\text { interior da } \\
\text { cobertura }\end{array}$} & \multicolumn{4}{|c|}{ IAF médio por classe de radiação } & \multirow{3}{*}{$\begin{array}{c}\text { IAF } \\
\text { Médio }\end{array}$} \\
\hline & I & II & III & IV & \\
\hline & \multicolumn{4}{|c|}{$(0-25 \%)(25-50 \%)(50-75 \%)(75-100 \%)$} & \\
\hline 0,0 & $\begin{array}{c}6,06 \\
(0,42)\end{array}$ & $\begin{array}{c}5,93 \\
(0,41)\end{array}$ & $\begin{array}{c}6,42 \\
(0,54)\end{array}$ & $\begin{array}{c}6,48 \\
(0,60)\end{array}$ & 6,32 \\
\hline 2,9 & $\begin{array}{c}5,54 \\
(0,36)\end{array}$ & $\begin{array}{c}5,40 \\
(0,46)\end{array}$ & $\begin{array}{c}5,91 \\
(0,57)\end{array}$ & $\begin{array}{c}5,91 \\
(0,70)\end{array}$ & 5,77 \\
\hline 4,9 & $\begin{array}{c}5,33 \\
(0,38)\end{array}$ & $\begin{array}{c}5,21 \\
(0,48)\end{array}$ & $\begin{array}{c}5,70 \\
(0,55)\end{array}$ & $\begin{array}{c}5,70 \\
(0,71)\end{array}$ & 5,57 \\
\hline 8,9 & $\begin{array}{c}5,50 \\
(0,39)\end{array}$ & $\begin{array}{c}5,19 \\
(0,44)\end{array}$ & $\begin{array}{c}5,66 \\
(0,59)\end{array}$ & $\begin{array}{c}5,53 \\
(0,66)\end{array}$ & 5,49 \\
\hline 12,9 & $\begin{array}{c}4,51 \\
(0,37)\end{array}$ & $\begin{array}{c}4,31 \\
(0,47)\end{array}$ & $\begin{array}{c}4,67 \\
(0,67)\end{array}$ & $\begin{array}{c}4,65 \\
(0,79)\end{array}$ & 4,57 \\
\hline 16,9 & $\begin{array}{c}3,60 \\
(0,43)\end{array}$ & $\begin{array}{c}3,49 \\
(0,46)\end{array}$ & $\begin{array}{c}3,82 \\
(0,79)\end{array}$ & $\begin{array}{c}3,54 \\
(1,21)\end{array}$ & 3,60 \\
\hline 20,9 & $\begin{array}{c}3,41 \\
(0,28)\end{array}$ & $\begin{array}{c}3,31 \\
(0,42)\end{array}$ & $\begin{array}{c}3,76 \\
(0,73)\end{array}$ & $\begin{array}{c}3,51 \\
(1,14)\end{array}$ & 3,52 \\
\hline
\end{tabular}

contraste com largas variações das estimativas isoladas em cada vertical e horário das medidas. No km 14, o IAF médio (última coluna de valores das tabelas) variou na faixa entre 6,32 e 6,52; no $\mathrm{km} 34$, o IAF médio ocorre no intervalo de 6,03 a 6,26. No conjunto de resultados obtidos para cada vertical e para cada horário, a faixa de variação espacial do IAF para o sítio experimental do $\mathrm{km} 14$ foi de 2,6 a 8,0 com um valor médio de 6,4; e para o sítio experimental do $\mathrm{km} 34$ essa faixa foi de $2,4 \mathrm{a}$ 8,0 com um valor médio de 6,1 . Essas largas variações precisam ser associadas a duas classes de fatores distintos: (i) condições externas da atmosfera que determinam as características do campo de radiação no topo da vegetação; (ii) estrutura e variabilidade natural da vegetação. Em geral, no primeiro caso, condições de céu limpo, com marcada predominância de radiação direta, são relevantes, necessárias e favoráveis ao estabelecimento da variabilidade espacial dos elementos vegetais no interior da cobertura, permitindo focalizar, em diferentes horários e a partir de um mesmo ponto de observação no interior da cobertura, diferentes zonas da cobertura com suas distribuições específicas de área foliar. Os eventos com predominância de radiação difusa determinam estimativas de área foliar que são praticamente independentes do horário da observação, pois para um mesmo ponto de observação as diferentes zonas da vegetação, com suas respectivas direções associadas, são ponderadas de forma igualitária na composição da atenuação da radiação em decorrência da isotropia aproximada do campo externo de radiação solar difusa. Esse comportamento da radiação solar no interior de floresta da região é comum entre os sítios já estudados, e a sua interpretação é inteiramente compatível com os elementos teóricos sobre o regime de radiação solar em meio vegetal, apresentados por Ross (1981) e Myneni et al. (1989).

Tabela 2 - Reserva do Cuieiras - ZF2, km 14; Segunda Vertical: 13 set -26 set 2001 . IAF calculado para cada classe de radiação, valores entre parênteses representam o desvio padrão.

\begin{tabular}{cccccc}
\hline \hline $\begin{array}{l}\text { Nível de } \\
\text { medida } \\
(\mathrm{m}) \text { no }\end{array}$ & \multicolumn{2}{c}{ IAF médio por classe de radiação } & \\
\cline { 2 - 5 } $\begin{array}{l}\text { interior da } \\
\text { cobertura }\end{array}$ & I & II & III & IV & $\begin{array}{c}\text { IAF } \\
\text { Médio }\end{array}$ \\
\cline { 2 - 5 } & $(0-25 \%)$ & $(25-50 \%)$ & $(50-75 \%)$ & $(75-100 \%)$ & \\
\hline 0,0 & 6,35 & 6,34 & 6,58 & 6,56 & 6,52 \\
& $(0,38)$ & $(0,60)$ & $(0,74)$ & $(0,82)$ & \\
2,7 & 5,95 & 5,83 & 6,05 & 6,20 & 6,09 \\
& $(0,36)$ & $(0,48)$ & $(0,66)$ & $(0,67)$ & \\
4,7 & 5,60 & 5,55 & 5,70 & 5,93 & 5,79 \\
& $(0,38)$ & $(0,47)$ & $(0,71)$ & $(0,66)$ & \\
8,7 & 4,70 & 4,72 & 4,90 & 5,03 & 4,93 \\
& $(0,37)$ & $(0,56)$ & $(0,81)$ & $(1,08)$ & \\
12,7 & 4,26 & 4,32 & 4,53 & 4,78 & 4,61 \\
& $(0,49)$ & $(0,50)$ & $(0,58)$ & $(0,69)$ & \\
16,7 & 3,17 & 3,33 & 3,67 & 3,99 & 3,75 \\
\hline \hline
\end{tabular}


Tabela 3 - Reserva do Cuieiras - ZF2, km14; Terceira Vertical: 26 set -10 out 2001 . IAF calculado para cada classe de radiação, valores entre parênteses representam o desvio padrão.

\begin{tabular}{|c|c|c|c|c|c|}
\hline \multirow{3}{*}{$\begin{array}{l}\text { Nível de } \\
\text { medida } \\
(\mathrm{m}) \text { no } \\
\text { interior da } \\
\text { cobertura }\end{array}$} & \multicolumn{4}{|c|}{ IAF médio por classe de radiação } & \multirow{3}{*}{$\begin{array}{c}\text { IAF } \\
\text { Médio }\end{array}$} \\
\hline & $\mathbf{I}$ & II & III & IV & \\
\hline & \multicolumn{4}{|c|}{$(0-25 \%)(25-50 \%)(50-75 \%)(75-100 \%)$} & \\
\hline 0,0 & $\begin{array}{c}5,65 \\
(0,32)\end{array}$ & $\begin{array}{c}6,17 \\
(0,50)\end{array}$ & $\begin{array}{c}6,40 \\
(0,81)\end{array}$ & $\begin{array}{c}6,56 \\
(0,78)\end{array}$ & 6,46 \\
\hline 2,7 & $\begin{array}{c}5,32 \\
(0,44)\end{array}$ & $\begin{array}{c}5,49 \\
(0,52)\end{array}$ & $\begin{array}{c}5,38 \\
(0,72)\end{array}$ & $\begin{array}{c}6,01 \\
(0,79)\end{array}$ & 5,90 \\
\hline 4,7 & $\begin{array}{c}5,11 \\
(0,39)\end{array}$ & $\begin{array}{c}5,19 \\
(0,51)\end{array}$ & $\begin{array}{c}5,55 \\
(0,69)\end{array}$ & $\begin{array}{c}5,78 \\
(0,72)\end{array}$ & 5,65 \\
\hline 8,7 & $\begin{array}{c}4,45 \\
(0,34)\end{array}$ & $\begin{array}{c}4,65 \\
(0,48)\end{array}$ & $\begin{array}{c}4,98 \\
(0,69)\end{array}$ & $\begin{array}{c}5,26 \\
(0,79)\end{array}$ & 5,11 \\
\hline 12,7 & $\begin{array}{c}4,21 \\
(0,38)\end{array}$ & $\begin{array}{c}4,37 \\
(0,59)\end{array}$ & $\begin{array}{c}4,80 \\
(0,69)\end{array}$ & $\begin{array}{c}5,08 \\
(0,84)\end{array}$ & 4,92 \\
\hline 16,7 & $\begin{array}{c}3,86 \\
(0,46)\end{array}$ & $\begin{array}{c}3,96 \\
(0,72)\end{array}$ & $\begin{array}{c}4,35 \\
(1,00)\end{array}$ & $\begin{array}{c}4,72 \\
(1,06)\end{array}$ & 4,53 \\
\hline 20,7 & $\begin{array}{c}3,94 \\
(0,36) \\
\end{array}$ & $\begin{array}{c}3,93 \\
(0,68) \\
\end{array}$ & $\begin{array}{c}4,46 \\
(0,80) \\
\end{array}$ & $\begin{array}{c}4,61 \\
(1,19) \\
\end{array}$ & 4,49 \\
\hline
\end{tabular}

Tabela 4 - Reserva do Cuieiras - ZF2, km 34; Primeira Vertical: 26 jul - 10 ago 2001. IAF calculado para cada classe de radiação, valores entre parênteses representam o desvio padrão.

\begin{tabular}{|c|c|c|c|c|c|}
\hline \multirow{3}{*}{$\begin{array}{l}\text { Nível de } \\
\text { medida } \\
(\mathrm{m}) \text { no } \\
\text { interior da } \\
\text { cobertura } \\
\end{array}$} & \multicolumn{4}{|c|}{ IAF médio por classe de radiação } & \multirow{3}{*}{$\begin{array}{c}\text { IAF } \\
\text { Médio }\end{array}$} \\
\hline & $\mathbf{I}$ & II & III & IV & \\
\hline & \multicolumn{4}{|c|}{$(0-25 \%)(25-50 \%)(50-75 \%)(75-100 \%)$} & \\
\hline 0,0 & $\begin{array}{c}6,21 \\
(0,32)\end{array}$ & $\begin{array}{c}6,09 \\
(0,36)\end{array}$ & $\begin{array}{c}6,20 \\
(0,58)\end{array}$ & $\begin{array}{c}6,40 \\
(0,65)\end{array}$ & 6,26 \\
\hline 2,7 & $\begin{array}{c}5,60 \\
(0,23)\end{array}$ & $\begin{array}{c}5,50 \\
(0,38)\end{array}$ & $\begin{array}{c}5,50 \\
(0,78)\end{array}$ & $\begin{array}{c}5,88 \\
(0,90)\end{array}$ & 5,72 \\
\hline 4,7 & $\begin{array}{c}5,17 \\
(0,20)\end{array}$ & $\begin{array}{c}5,13 \\
(0,34)\end{array}$ & $\begin{array}{c}5,14 \\
(0,61)\end{array}$ & $\begin{array}{c}5,62 \\
(0,72)\end{array}$ & 5,40 \\
\hline 8,7 & $\begin{array}{c}4,45 \\
(0,28)\end{array}$ & $\begin{array}{c}4,46 \\
(0,38)\end{array}$ & $\begin{array}{c}4,46 \\
(0,60)\end{array}$ & $\begin{array}{c}5,09 \\
(0,86)\end{array}$ & 4,80 \\
\hline 12,7 & $\begin{array}{c}3,72 \\
(0,22)\end{array}$ & $\begin{array}{c}3,61 \\
(0,44)\end{array}$ & $\begin{array}{c}3,62 \\
(0,78)\end{array}$ & $\begin{array}{c}4,42 \\
(1,09)\end{array}$ & 4,06 \\
\hline 16,7 & $\begin{array}{c}3,00 \\
(0,22)\end{array}$ & $\begin{array}{c}2,85 \\
(0,45)\end{array}$ & $\begin{array}{c}2,72 \\
(0,84)\end{array}$ & $\begin{array}{c}3,38 \\
(1,27)\end{array}$ & 3,12 \\
\hline 20,7 & $\begin{array}{c}2,80 \\
(0,26)\end{array}$ & $\begin{array}{c}2,46 \\
(0,60)\end{array}$ & $\begin{array}{c}2,44 \\
(0,75)\end{array}$ & $\begin{array}{c}2,75 \\
(1,35)\end{array}$ & 2,65 \\
\hline
\end{tabular}

Os desvios padrões da função A(z) em cada vertical e entre as classes de radiação solar mostram comportamentos já conhecidos de outros sítios experimentais na região (Marques Filho \& Dallarosa 2000,2001): em geral, esses desvios são marcadamente crescentes, na ordem, desde a classe I até a classe IV. No km 14, os valores médios da função A(z) variam numa faixa menor que 0,5 para todas as alturas inferiores a 15
Tabela 5 - Reserva do Cuieiras - ZF2, km 34; Segunda Vertical: 15 ago - 21 ago 2001 IAF calculado para cada classe de radiação, valores entre parênteses representam o desvio padrão.

\begin{tabular}{|c|c|c|c|c|c|}
\hline \multirow{3}{*}{$\begin{array}{l}\text { Nível de } \\
\text { medida } \\
(\mathrm{m}) \text { no } \\
\text { interior da } \\
\text { cobertura }\end{array}$} & \multicolumn{4}{|c|}{ IAF médio por classe de radiação } & \multirow{3}{*}{$\begin{array}{c}\text { IAF } \\
\text { Médio }\end{array}$} \\
\hline & I & II & III & IV & \\
\hline & \multicolumn{4}{|c|}{$(0-25 \%)(25-50 \%)(50-75 \%)(75-100 \%)$} & \\
\hline 0,0 & & $\begin{array}{c}5,81 \\
(0,32)\end{array}$ & $\begin{array}{c}5,88 \\
(0,57)\end{array}$ & $\begin{array}{c}6,09 \\
(0,94)\end{array}$ & 6,03 \\
\hline 2,6 & & $\begin{array}{c}5,03 \\
(0,30)\end{array}$ & $\begin{array}{c}5,17 \\
(0,46)\end{array}$ & $\begin{array}{c}5,47 \\
(0,76)\end{array}$ & 5,37 \\
\hline 4,6 & & $\begin{array}{c}4,27 \\
(0,30)\end{array}$ & $\begin{array}{c}4,50 \\
(0,52)\end{array}$ & $\begin{array}{c}4,76 \\
(0,87)\end{array}$ & 4,66 \\
\hline 8,6 & & $\begin{array}{c}3,62 \\
(0,33)\end{array}$ & $\begin{array}{c}4,11 \\
(0,63) .\end{array}$ & $\begin{array}{c}4,34 \\
(0,98)\end{array}$ & 4,22 \\
\hline 12,6 & & $\begin{array}{c}3,32 \\
(0,38)\end{array}$ & $\begin{array}{c}3,78 \\
(0,72) .\end{array}$ & $\begin{array}{c}3,80 \\
(1,06)\end{array}$ & 3,74 \\
\hline 16,6 & & $\begin{array}{c}3,36 \\
(0,38)\end{array}$ & $\begin{array}{c}3,67 \\
(0,70)\end{array}$ & $\begin{array}{c}3,70 \\
(1,00)\end{array}$ & 3,66 \\
\hline 20,6 & & $\begin{array}{c}2,76 \\
(0,44) \\
\end{array}$ & $\begin{array}{c}3,26 \\
(0,90) \\
\end{array}$ & $\begin{array}{c}3,15 \\
(1,37) \\
\end{array}$ & 3,13 \\
\hline
\end{tabular}

Tabela 6 - Reserva do Cuieiras ZF2, Km34; Terceira Vertical: 11 out - 21 nov 2001 IAF calculado para cada classe de radiação, valores entre parênteses representam o desvio padrão.

\begin{tabular}{|c|c|c|c|c|c|}
\hline \multirow{3}{*}{$\begin{array}{l}\text { Nível de } \\
\text { medida } \\
(\mathrm{m}) \text { no } \\
\text { interior da } \\
\text { cobertura }\end{array}$} & \multicolumn{4}{|c|}{ IAF médio por classe de radiação } & \multirow{3}{*}{$\begin{array}{c}\text { IAF } \\
\text { Médio }\end{array}$} \\
\hline & I & II & III & IV & \\
\hline & \multicolumn{4}{|c|}{$(0-25 \%)(25-50 \%)(50-75 \%)(75-100 \%)$} & \\
\hline 0,0 & $\begin{array}{c}6,06 \\
(0,36)\end{array}$ & $\begin{array}{c}6,00 \\
(0,43)\end{array}$ & $\begin{array}{c}6,10 \\
(0,66)\end{array}$ & $\begin{array}{c}6,25 \\
(0,84)\end{array}$ & 6,15 \\
\hline 2,3 & $\begin{array}{c}5,73 \\
(0,37)\end{array}$ & $\begin{array}{c}5,68 \\
(0,43)\end{array}$ & $\begin{array}{c}5,79 \\
(0,64)\end{array}$ & $\begin{array}{c}5,99 \\
(0,85)\end{array}$ & 5,86 \\
\hline 4,3 & $\begin{array}{c}5,47 \\
(0,37)\end{array}$ & $\begin{array}{c}5,38 \\
(0,45)\end{array}$ & $\begin{array}{c}5,49 \\
(0,67)\end{array}$ & $\begin{array}{c}5,73 \\
(0,84)\end{array}$ & 5,59 \\
\hline 8,3 & $\begin{array}{c}5,28 \\
(0,33)\end{array}$ & $\begin{array}{c}5,17 \\
(0,47)\end{array}$ & $\begin{array}{c}5,21 \\
(0,66)\end{array}$ & $\begin{array}{c}5,32 \\
(0,92)\end{array}$ & 5,26 \\
\hline 12,3 & $\begin{array}{c}5,29 \\
(0,33)\end{array}$ & $\begin{array}{c}5,13 \\
(0,48)\end{array}$ & $\begin{array}{c}5,14 \\
(0,74)\end{array}$ & $\begin{array}{c}5,15 \\
(1,01)\end{array}$ & 5,16 \\
\hline 16,3 & $\begin{array}{c}3,99 \\
(0,33)\end{array}$ & $\begin{array}{c}3,95 \\
(0,52)\end{array}$ & $\begin{array}{c}4,02 \\
(0,90)\end{array}$ & $\begin{array}{c}4,05 \\
(1,31)\end{array}$ & 4,02 \\
\hline 20,3 & $\begin{array}{c}3,97 \\
(0,40)\end{array}$ & $\begin{array}{c}3,76 \\
(0,45)\end{array}$ & $\begin{array}{c}3,93 \\
(0,78)\end{array}$ & $\begin{array}{c}4,01 \\
(1,18)\end{array}$ & 3,95 \\
\hline
\end{tabular}

$\mathrm{m}$, indicando uma regularidade horizontal da cobertura nesta zona; em contraposição, o sítio do km 34 apresenta larga variação dos perfis na zona indicada acima e em toda a extensão das verticais.

Nas curvas médias de distribuição vertical de área foliar, obtidas no presente estudo (Figuras 2 e 3), como já encontrado para outras coberturas na Amazônia (Marques Filho \& Dallarosa 


\section{ACTA AMAZONICA}

2000,2001), existem pelo menos duas zonas distintas de concentração de área foliar: uma principal, situada na parte alta da vegetação, e pelo menos uma secundária, na parte baixa da vegetação. Essas zonas são intercaladas por uma zona de transição com baixa densidade de área foliar.

$\mathrm{Na}$ figura $2(\mathrm{~km} \mathrm{14})$, existem duas zonas distintas de concentração de área foliar: uma principal situada na parte alta da vegetação, com concentração máxima de área foliar de 0,34 $\mathrm{m}^{2} . \mathrm{m}^{-3}$, situada logo acima de $27 \mathrm{~m}$ de altura; e uma secundária na parte baixa da vegetação, que apresenta picos de densidade de área foliar variando entre 0,16 a $0,25 \mathrm{~m}^{2} \cdot \mathrm{m}^{-3}$. Esses dois estratos são intercalados por uma zona de transição com baixa densidade de área foliar, cujo valor mínimo atinge $0,12 \mathrm{~m}^{2} \cdot \mathrm{m}^{-}$ 3 , localizada na faixa de 18 a $20 \mathrm{~m}$.

Na Figura 3 (km 34), a curva média de área foliar apresenta três estratos de vegetação: a zona principal à qual correspondem picos de concentração de área foliar da ordem de $0,33 \mathrm{~m}^{2} \cdot \mathrm{m}^{-3}$ situada entre 26 e $28 \mathrm{~m}$ de altura; a segunda zona apresenta densidade média máxima de área foliar de $0,24 \mathrm{~m}^{2} \cdot \mathrm{m}^{-3}$ na altura de $15 \mathrm{~m}$; e finalmente uma zona próxima ao solo com pico de densidade média de área foliar de $0,26 \mathrm{~m}^{2} \cdot \mathrm{m}^{-3}$ na altura de $4 \mathrm{~m}$. Essa zona corresponde à existência de alta densidade de plântulas e arvoretas especificamente nesse local. Os dois estratos superiores são intercalados por zona de transição localizada na faixa de 17 a $20 \mathrm{~m}$ com densidade de área foliar mínima de $0,13 \mathrm{~m}^{2} \cdot \mathrm{m}^{-3}$. A segunda zona de transição ocorre no intervalo de 6 a $10 \mathrm{~m}$ com uma densidade de área foliar mínima de $0,15 \mathrm{~m}^{2} \cdot \mathrm{m}^{-3}$.

Sobre o estrato superior da vegetação nesses dois sítios experimentais, é preciso observar que eles são constituídos pelas copas das grandes árvores combinadas com as copas de árvores de médio porte, eventualmente superpostas, formando zonas da vegetação com folhagem densa. A inexistência de medidas acima de $22 \mathrm{~m}$ impede um detalhamento de eventuais variações de área foliar nesse estrato; esse fato tem pouco

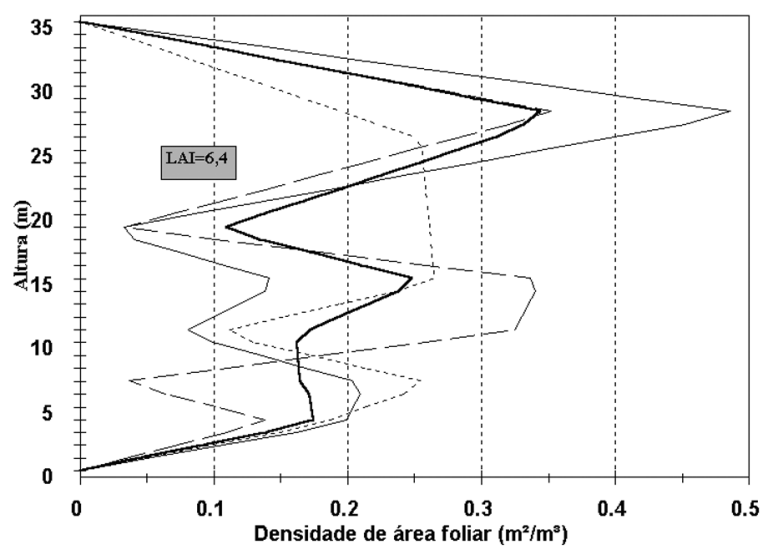

Figura 2 - Representa a densidade de área foliar das três verticais e a densidade média de área foliar da ZF2-km 14: ( _ _ - ) primeira vertical; (----) segunda vertical; (-) terceira vertical; (_-) densidade média de área foliar. efeito sobre a forma da função a(z), especialmente em seus valores médios, pois a zona superior da vegetação é mais homogênea e densa.

Amaral et al. (2002), com o objetivo de estudar a composição florística do sub-bosque do sítio experimental do $\mathrm{km} 34$, fizeram um inventário de 0,05 hectare de floresta. As plantas foram divididas em quatro classes de tamanho: plântula (altura (h) $<0,5 \mathrm{~m})$, muda $1(0,5 \mathrm{~m}<\mathrm{h}<1,5 \mathrm{~m})$, muda $2(1,5$ $\mathrm{m}<\mathrm{h}<3,0 \mathrm{~m}$ ) e estabelecida $(\mathrm{h}>3,0 \mathrm{~m})$. Os dados evidenciaram maior representatividade da classe plântula correspondendo a $61,7 \%$ do total de plantas e o menor percentual foi observado na classe estabelecida com $4,4 \%$; os demais percentuais foram distribuídos entre as classes muda 1 com $26,5 \%$ e muda 2 com 7,4\%. Isto justifica o pico de densidade média de área foliar localizado na faixa de 0 a $5 \mathrm{~m}$, que corresponde ao terceiro estrato encontrado nesse sítio experimental.

\section{Comparação de resultados de diferentes sítios experimentais}

As variações espaciais entre verticais de um mesmo sítio experimental poderiam sugerir a ocorrência de estruturas de vegetação distintas. Guillaumet \& Kahn (1982) estudaram a estrutura e a dinâmica da floresta na ZF2, relacionando as possíveis variações principalmente às conformações topográficas sobre as quais desenvolve-se a vegetação. Nesse estudo, as variações localizadas de distribuição de folhas são imputadas preferencialmente às peculiaridades do solo e à dinâmica interna da própria vegetação, que determinam crescimentos diferenciados de árvores em resposta combinada com o regime luminoso predominante em cada local. Os intercâmbios de massa e de energia entre coberturas vegetais e a atmosfera integram e são dirigidos por características médias

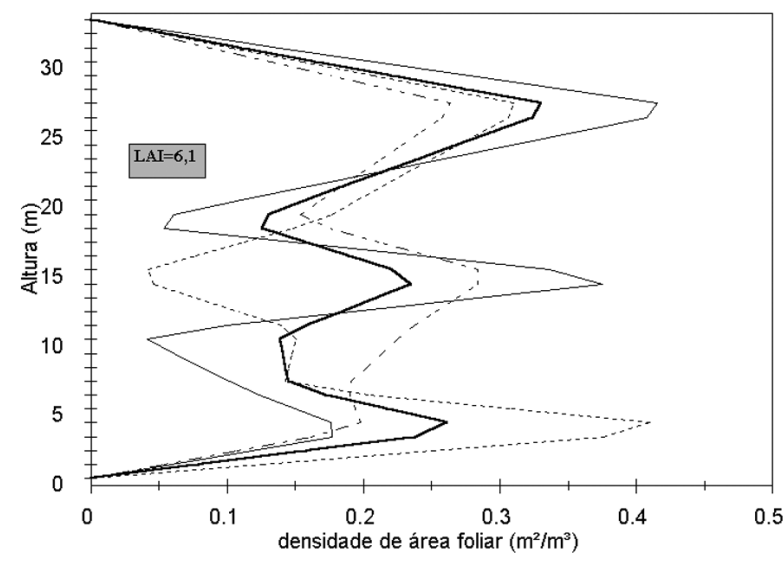

Figura 3 - Representa a densidade de área foliar das três verticais e a densidade média de área foliar da ZF2-km 34: ( - primeira vertical; (----) segunda vertical; (-) terceira vertical; ( $(-)$ densidade média de área foliar. 


\section{ACTA \\ AMAZONICA}

RADIACÃO SOLAR E DISTRIBUIČ̃̃O VERTICAL DE ÁREA FOLIAR EM FLORESTA - RESERVA BIOLÓGICA DO CUIEIRAS - ZF2, MANAUS da vegetação, dentre as quais a distribuição vertical de área foliar tem uma contribuição dominante. Como essa função varia e quais aspectos a ela associados são comuns para diferentes locais na região Amazônica, constituem questionamentos fundamentais na busca de resultados que sejam de aplicação mais geral. Nesse sentido, os conceitos de estrutura da vegetação e as metodologias adotadas para caracteriza-la, conforme apresentados e discutidos no estudo de Guillaumet \& Kahn (1982), constituem um cenário adequado a ser combinado com estudos do regime de radiação no interior da vegetação, para compor eventuais esquemas genéricos de distribuição vertical de área foliar. No contexto mais geral de intercâmbios de massa e de energia entre coberturas vegetais e a atmosfera, Araújo et al. (2002) abordaram os fatores externos à vegetação (relevo e estabilidade atmosférica) e estabeleceram, pela aplicação de algoritmos apropriados, a extensão horizontal de vegetação em torno dos pontos de medidas (torres micrometeorológicas), que contribui para a formação de $80 \%$ dos fluxos. Essa operação integra as contribuições para os fluxos, indistintamente, de vegetação dos platôs, das encostas e dos baixios. Um desafio teórico e experimental importante consiste em combinar essas condições externas de estabilidade atmosférica e as características de relevo dos sítios experimentais com as distribuições verticais de área foliar, para a construção de um algoritmo aperfeiçoado que defina a representatividade de medidas pontuais de fluxos de massa $\mathrm{e}$ de energia entre vegetação e atmosfera.
Nesta etapa de comparação entre curvas médias de densidade de área foliar para coberturas vegetais situadas na Amazônia, os sítios e os períodos de observação incluídos na presente análise são os seguintes:

1 - Reserva florestal Ducke ( $\left.02^{\circ} 56^{\prime} \mathrm{S} ; 59^{\circ} 57^{\prime} \mathrm{W}\right)$ - 29 out - $11 \mathrm{dez}$ de 1998.

2 - Reserva Biológica Jaru ( $\left(10^{\circ} 05^{\prime} \mathrm{S} ; 61^{\circ} 55^{\prime} \mathrm{W}\right)$ - 30 out - 24 nov de 1999.

3 - Reserva Biológica do Cuieiras, km14, 02 $35^{\circ} \mathrm{S} ; 60^{\circ} 06^{\prime} \mathrm{W} ; 26$ jul - 21 nov 2001.

4- Reserva Biológica do Cuieiras, $\mathrm{km} 34,02^{\circ} 36^{\prime} \mathrm{S} ; 60^{\circ} 12^{\prime} \mathrm{W} ; 26$ jul-21 nov 2001.

As distribuições médias de área foliar para esses quatro sítios são reproduzidas nas figuras 4 (a), (b), (c) e (d). As características principais e as respectivas faixas de variações desse conjunto de coberturas são as seguintes:

1 - Índice de área foliar total: varia entre 5,6 e 6,4.

2 -Densidade máxima de área foliar na zona principal superior: 0,27 a $0,34 \mathrm{~m}^{2} \cdot \mathrm{m}^{-3}$.

3 - Densidade mínima na zona de transição: $0,10 \mathrm{~m}^{2} \cdot \mathrm{m}^{-3}$ a 0,14 $\mathrm{m}^{2} \cdot \mathrm{m}^{-3}$.

4 - Localização da zona de transição: 15 m a $20 \mathrm{~m}$.
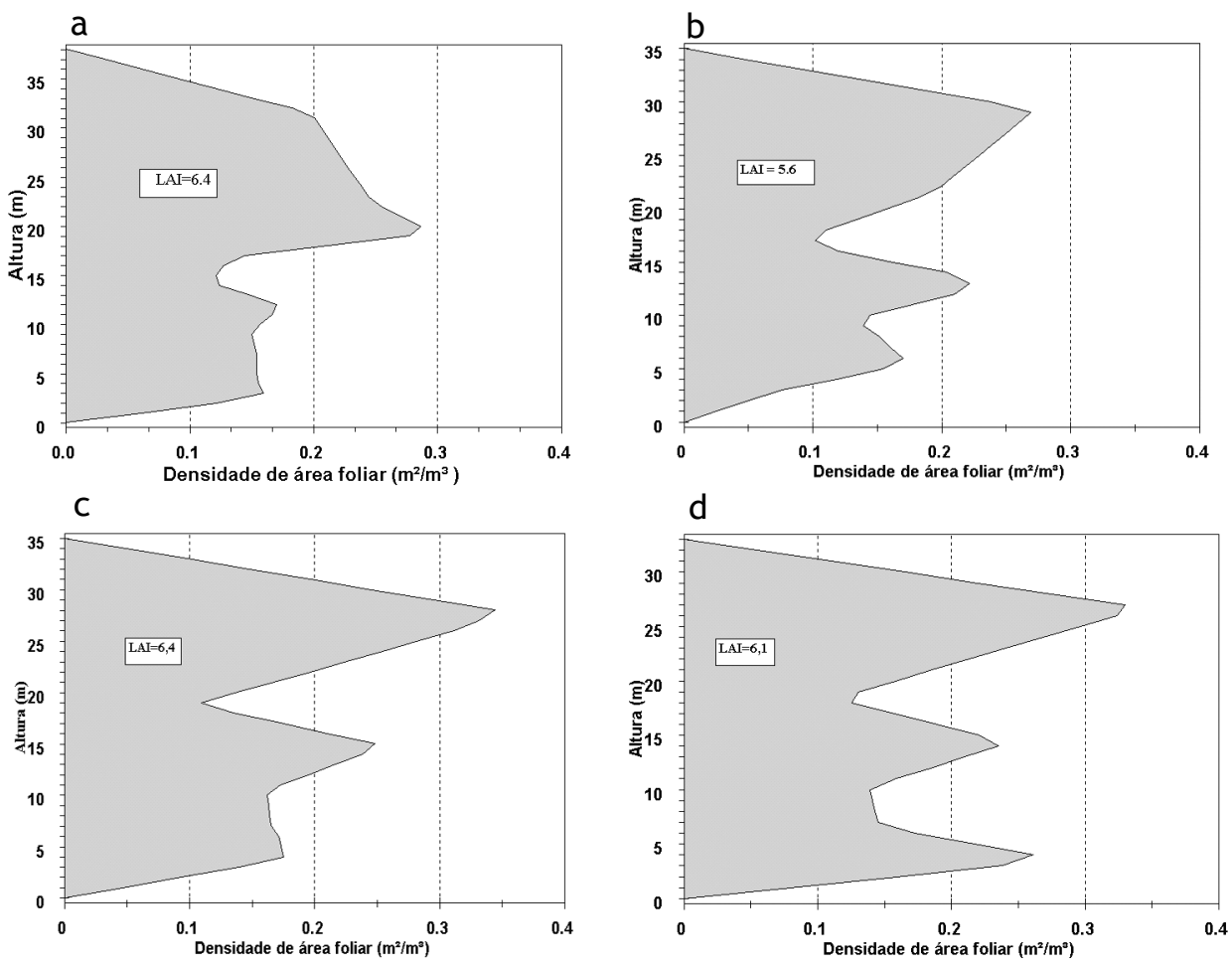

Figura 4 - Distribuição vertical de área foliar para quatro sítios experimentais da região Amazônica: (a) Reserva Ducke (Marques Filho \& Dallarosa,2000); (b) Reserva Jaru (Marques Filho \& Dallarosa,2001): (c) Reserva Cuieiras, ZF2 - Km14: (d) Reserva Cuieiras, ZF2 - Km34. 


\section{ACTA \\ AMAZONICA}

RADIAÇÃO SOLAR E DISTRIBUIÇÃO VERTICAL DE ÁREA FOLIAR EM

FLORESTA - RESERVA BIOLÓGICA DO CUIEIRAS - ZF2, MANAUS
5 - Altura média da vegetação; $33 \mathrm{~m}$ a $38 \mathrm{~m}$.

6 - Estratificação: 2 a 3 estratos.

Comparando os perfis de área foliar dos quatro sítios experimentais (reservas Ducke, Jaru e Cuieiras, km 14 e km 34), verifica-se uma pequena variação no total de área foliar entre os sítios, em contraste aos aspectos qualitativos muito distintos, com traços singulares em cada ponto experimental, que bem sugerem a ocorrência de estrutura da vegetação com padrões localizados. Um aspecto comum a todos os perfis é a existência de uma zona principal de concentração de área foliar na parte superior da vegetação situada acima da altura de $20 \mathrm{~m}$, que incorpora pelo menos $50 \%$ da área foliar total da cobertura; em seguida ocorre uma zona de transição com baixa densidade de área foliar e situada nos diferentes perfis na faixa entre $15 \mathrm{e}$ $20 \mathrm{~m}$. Abaixo dessa zona, tem-se a parte inferior da vegetação que pode se apresentar em sua distribuição média de área foliar desde uma forma constante (perfil da Reserva Ducke) até o caso extremo dessa zona secundária subdividida de forma clara em dois estratos (perfil da ZF2- km 34). Os dois casos intermediários apresentam picos isolados bastante próximos, sugerindo a presença de um único estrato inferior com densidade de área foliar ligeiramente variável (perfis da Reserva Jaru e da ZF2 - km 14),

Esses quatro perfis foram determinados pelo mesmo aparato experimental (reticulado móvel de sustentação de tubos solarímetros) e via de regra compostos a partir de medidas de radiação solar em três verticais distintas, acima e no interior de cada cobertura vegetal, em períodos contínuos que variam entre 10 e 20 dias. A variabilidade de formas de perfis entre as verticais de um mesmo sítio experimental parece indicar a tendência para iniciativas de aperfeiçoamento do aparato de medidas, qual seja, um conjunto simultâneo de medidas em verticais próximas com adensamento de sensores nas zonas mais complexas da estrutura da vegetação.

À luz desses resultados experimentais disponíveis, as características apresentadas pelas funções a $(\mathrm{z})$, relacionadas acima (1-6), e as faixas de valores em que elas ocorrem, já permitem e devem ser considerados em propostas de formas adequadas para a função a(z), usadas em estudos de modelagem de interações entre clima e vegetação da região.

\section{CONCLUSÃO}

A função a(z) de densidade de área foliar, estabelecida no presente estudo para dois sítios experimentais da ZF2, apresenta-se no km 14 com dois estratos principais: um estrato superior associado às copas das árvores que se situam acima de $20 \mathrm{~m}$ e um estrato inferior referente às árvores com copas situadas abaixo dessa altura; para o $\mathrm{km} 34$ o estrato superior da vegetação é similar aquele da vegetação do km14, em contraste com a parte inferior da cobertura que aparece subdividida em dois estratos com picos de área foliar bem definidos. Isto representa a distribuição vertical média de área foliar da floresta de Cuieiras e associa o valor de 6,4 como o índice de área foliar (IAF) para a cobertura do $\mathrm{km} 14 \mathrm{e} \mathrm{6,1}$ para a cobertura do $\mathrm{km} 34$.
A comparação das características principais dos perfis de área foliar de quatro sítios experimentais situados na região revela uma acentuada convergência quantitativa entre as coberturas vegetais em foco (IAF varia entre 5,6 e 6,4). Em contraposição, os aspectos qualitativos desses perfis são distintos e reforçam a diversidade natural de estruturas de vegetação, que podem apresentar até uma estratificação adicional na parte inferior da vegetação junto ao solo, decorrentes de uma alta densidade local de plântulas e arvoretas.

\section{AGRADECIMENTOS}

Os autores agradecem aos técnicos da Coordenação de Pesquisas em Geociências do INPA: Afonso Ligório da Mota, Hermes Braga Xavier, Jauapery Neves Pereira Júnior, Sérgio Magno Valério de Souza e Valdelice Pereira da Silva pela ajuda na preparação e instalação dos sistemas de medidas, aos pesquisadores Dr. Antônio Donato Nobre e Alessandro Carioca de Araújo pelo apoio; ao CNPq/LBA pela bolsa DTI concedida a Vanusa Bezerra Pachêco. Este estudo se insere no Projeto de Pesquisa: "Balanço de energia, vapor d'água e $\mathrm{CO}_{2}$ em área de floresta tropical na Amazônia Central", financiado sob a referência PPD 95/0966.

\section{BIBLIOGRAFIA CITADA}

Amaral, I. L.; Oliveira, A. N.; Nobre, A. D. 2002. Florística em subbosque de floresta ombrófila densa de terra firme na Amazônia Central, Amazonas, Brasil, $2^{\text {a }}$. Conferência Científica Internacional do LBA, Manaus, Amazonas, julho 2002.

Andrieu, B.; Baret, F. 1993. Indirect methods of estimating crop structure from optical measurements. In: VarletGrancher, C.; Bonhomme, R.; Sinoquet, H. (Eds.) 1993 Crop Structure and Light Microclimate. INRA, France. p. 285-322.

Araújo, A. C.; Nobre, A. D.;Kruijt, B.; Elbers, J. A.; Dallarosa, R.; Stefani, P.; von Randow, C.; Manzi, A. O.; Culf, A. D.; Gash, H. C.; Valentini, R.; Kabat, P.; 2002. Comparative measurements of carbon dioxide fluxes from two nearby towers in a central Amazonian rainforest: The Manaus LBA site. Journal of Geophysical Research, 107(D20), 8090. LBA58:1-20.

Chauvel, A.1982. Os latossolos amarelos, álicos, argilosos dentro dos ecossistemas das bacias experimentais do INPA e da região vizinha. Acta Amazonica, Suplemento 12(3): 47-60.

Guillaumet, J. L.; Kahn, F. 1982. Structure et dynamisme de la forêt. Acta Amazonica, Suplemento 12(3): 61-77.

Honzák, M.; Lucas, R.M.; Amaral. I.; Curran, P.J.; Foody, G.M.; Amaral, S., 1996. Estimation of the leaf area index and total biomass of tropical regenerating forests: comparison of methodologies, in: Gash, J.H.C.; Nobre, C.A.; Roberts, J.M.; Victoria, R.L. (Eds.) 1996. Amazonian Deforestation and Climate. Institute of Hydrology, UK. p, 365-381. 


\section{ACTA AMAZONICA}

RADIAÇÃO SOLAR E DISTRIBUIÇÃO VERTICAL DE ÁREA FOLIAR EM FLORESTA - RESERVA BIOLÓGICA DO CUIEIRAS - ZF2, MANAUS
Jardim, F.C.S.; Hosokawa, R.T. 1986/1987. Estrutura da floresta equatorial úmida da Estação Experimental de Silvicultura Tropical do INPA. Acta Amazonica, 16/17(único):411-508

Marques Filho, A.O. 1992. Modèles des transferts radiatifs à l'intérieur des couverts végétaux - les solutions analytiques. Acta Amazonica, 22(4):541-565.

Marques Filho, A.O. 1997. Regime de radiação solar e características da vegetação - Modelos de inversão. Acta Amazonica, 27(2):119-134.

Marques Filho, A.O.; Dallarosa, R.G. 2000. Interceptação de radiação solar e distribuição espacial de área foliar em floresta de terra firme da Amazônia Central. Acta Amazonica, 30(3):453-470.

Marques Filho, A.O.; Dallarosa, R.G. 2001. Atenuação de radiação solar e distribuição vertical de área foliar em floresta - Reserva Jaru, Rondônia, Brasil. Acta Amazonica, 31(1):39-59.

McWilliam, A.L.C.; Roberts, J.M.; Cabral, O.M.R.; Leitão, M.V.B.R.; Costa, A.C.L.; Maitelli, G.T.; Zamparoni, C.A.G.P. 1993. Leaf area index and above ground biomass of terra firme rain forest and adjacent clearings in Amazonia. Functional Ecology, 7: 210-217.

Myneni, R.R.; Ross, J.; Asrar, G. 1989. A review on the theory of photon transport in plant canopies. Agricultural and Forest Meteorology, 45: 1-153.
Nilson, T. 1971. A theoretical analysis of the frequency of gaps in plant stands, Agricultural and Forest Meteorology, 8: 2538.

Oliveira, A. N.; Amaral, I. L.; Nobre, A. D.; Couto, L. B.; Sato, R. M.;Santos, J. L.; Ramos, J. 2002. Composição e diversidade florística de uma floresta ombrófila densa de terra firme na Amazônia Central, Amazonas, BRASIL, 2a. Conferência Científica Internacional do LBA, Manaus/AM, julho 2002.

Ross, J. 1981. The Radiation Regime and the Architecture of Plant Stands. Dr. W. Junk Publ., The Netherlands. 391p.

Wandelli, E.V.; Marques Filho, A.O. 1999. Medidas de radiação solar e índice de área foliar de coberturas vegetais. Acta Amazonica, 29(1): 57-78.

Welles, J.M.; Norman, J.M. 1991. Instrument for indirect measurement for canopy architecture. Agronomy Journal, 53:818-825.

\section{RECEBIDO EM 05/08/2002 ACEITO EM 20/10/2005}

